\title{
AVALIAÇÕES HISTOMÉTRICA, RADIOGRÁFICA E IMUNOLÓGICA DOS TECIDOS PERIODONTAIS DE RATAS OVARIECTOMIZADAS COM PERIODONTITE INDUZIDA
}

\author{
HISTOMETRICAL, RADIOGRAPHIC AND IMMUNOLOGICAL EVALUATIONS OF THE PERIODONTAL \\ TISSUES OF FEMALE OVARIECTOMIZED RATS WITH INDUCED PERIODONTITIS
}

\author{
Carolina de Cássia Boamorte1; Lais Fernanda Pasqualotto²; Ana Claudia Paiva Alegre Maller³ Patricia Oehlmeyer Nassar; \\ Sara Cristina Sagae5; Rose Meire Costa Brancalhão6; Lucinéia de Fátima Chasko Ribeiro7; Carlos Augusto Nassar ${ }^{8}$ \\ 1 - Mestre em Biociências e Saúde, Universidade Estadual do Oeste do Paraná (UNIOESTE), Cascavel, Paraná. \\ 2 - Mestranda em Biociências e Saúde, UNIOESTE, Cascavel, Paraná. \\ 3 - Pós-Doutoranda no Programa Strictu Sensu em Biociências e Saúde, UNIOESTE, Cascavel, Paraná. \\ 4 - DDS em Periodontia. Professora Adjunta de curso de Odontologia e Programas de Pós-Graduação em Biociências, Saúde e Odontologia da \\ UNIOESTE, Cascavel, Paraná. \\ 5 - DDS em Biologia Funcional e Molecular. Professora Adjunta no Centro de Ciências Médicas e Farmacêuticas da UNIOESTE, Cascavel, Paraná. \\ 6 - Doutora em Zoologia. Professora associada da disciplina de Biologia Celular e do Programa de Pós-Graduação em Biociências e Saúde da \\ UNIOESTE, Cascavel, Paraná. \\ 7 - Doutora em Biologia Celular e Molecular. Professora adjunta da disciplina de Anatomia Humana e do Programa de Pós-Graduação em \\ Biociências e Saúde da UNIOESTE, Cascavel, Paraná. \\ 8 - DDS em Periodontia. Professor Associado de curso de Odontologia e Programas de Pós-Graduação em Biociências, Saúde e Odontologia da \\ UNIOESTE, Cascavel, Paraná.
}

\section{RESUMO}

Objetivo: A relação da osteoporose com pós-menopausa é controversa, mas pode ser um fator de risco para doença periodontal. Assim o objetivo deste projeto foi avaliar a influência da deficiência de estrógeno nos tecidos periodontais de ratas ovariectomizadas com peridontite induzida. Material e método: Quarenta ratas com 8 semanas de vida foram divididas aleatoriamente em 4 grupos: 1) Grupo controle; 2) Grupo ligadura; 3) Grupo ovariectomia; 4) Grupo ovariectomia e ligadura; sendo que todos os animais receberam dieta padrão e água à vontade. Aos 70 dias de vida os animais foram anestesiados e foi realizada a indução da doença periodontal, sendo após este procedimento aos 79 dias de vida, as ratas também foram submetidas à anestesia e à cirurgia de ovariectomia. Aos 100 dias de vida, as ratas foram sacrificadas sendo retiradas as hemimandíbulas esquerda e direita de cada rata para análises histomorfométrica e radiográfica, bem como amostras do tecido gengival para avaliar os tecidos periodontais. Resultados: Os resultados demonstraram que na análise radiográfica e histomorfométrica do osso alveolar dos primeiros molares inferiores nos grupos onde foi feita a indução da doença periodontal por ligadura, houve maior perda óssea alveolar significativamente $(p<0.05)$. Em relação às concentrações de citocinas, os grupos que foram ovariectomizadas apresentaram concentrações maiores estatisticamente significante em relação aos demais $(\mathrm{p}<0.05)$. Conclusão: Assim pode-se concluir que a deficiência de estrógeno poderia contribuir para uma evolução mais rápida da perda óssea alveolar em ratas ovarietomizadas.

Palavras-chaves: Periodontite; menopausa; osteoporose; ovariectomia

\section{INTRODUÇÃO}

A menopausa, referida como à última menstruação, é um período de inatividade reprodutiva, que confere às mulheres queda na produção hormonal, principalmente do estradiol, impedindo a proliferação do endotélio. Tal ciclo do sistema reprodutor feminino, também conhecido por climatério traz consequências biológicas, psicológicas e sociais ${ }^{1}$. Uma das consequências da menopausa é a redução da produção estrogênica, uma vez que o estrógeno é responsável por um conjunto de funções, fora o eixo reprodutivo, como a modulação do metabolismo ósseo e mineral, cardíaco e função vascular, cognição e da memória, entre outros².

Posteriormente a menopausa, as consequências biológicas aparecem na forma de algumas doenças devido às alterações hormonais, que entre essas, são responsáveis pela diminuição da massa óssea, caracterizando as mulheres uma susceptibilidade 
a desenvolver osteoporose ${ }^{3}$. Essa doença óssea - metabólica, considerada uma disfunção sistêmica, altera a microarquitetura dos ossos, inclusive os da face ${ }^{4}$.

A osteoporose, após a menopausa, está diretamente relacionada ao hipoestrogenismo, acarretando aceleração da atividade de reabsorção e aumentand o o turn-over desse tecido, levando a uma perda óssea excessiva, atingindo ossos da maxila, mandíbula e ossos longos. A osteoporose tem sido evidenciada e sua correlação com a periodontia pode ser devido à diminuição na densidade da massa óssea esquelética, massa óssea alveolar interproximal e a perda de inserção clínica em pacientes com doença periodontal ${ }^{5}$.

Outra doença que afeta grande proporção da população é a periodontite, com sua natureza polimicrobiana orquestra um mecanismo complexo de inflamação que é caracterizado por destruição das fibras colágenas, outros constituintes da matriz do ligamento periodontal e do osso alveolar, em conjunto com a formação de bolsas periodontais ${ }^{6,7}$. É causada predominantemente por bactérias que liberam endotoxinas que ativam citocinas pró-inflamatórias (interleucina 1 - IL1; fator de necrose tumoral alfa - TNF- $\alpha$; entre outras) que afetam os tecidos de suporte dos dentes ${ }^{8-11}$.

Lopes et al. ${ }^{12}$ (2008) e Passos et al. ${ }^{13}$ (2010) concluíram, que o conhecimento atual sobre a osteoporose pós-menopausa ser um fator de risco para a doença periodontal, ainda é insuficiente e requer mais estudos sobre o tema e também sobre os reais mecanismos biológicos que relacionam essas evidências.

Há controvérsias em estudos na relação da osteoporose na pós-menopausa como sendo um fator de risco para doença periodontal ${ }^{14,15}$. Bem como, tem se estudado nas últimas décadas os efeitos dos fitoestrógenos, apesar de não ter sido descrito claramente qual seu mecanismo de ação ${ }^{16}$. Logo, o objetivo deste estudo foi avaliar a influência da deficiência de estrógeno nos tecidos periodontais de ratas ovariectomizadas com peridontite induzida.

\section{MATERIAL E MÉTODO}

\section{Animais}

Foram utilizadas 40 ratas Wistar (peso corporal entre 180$350 \mathrm{~g}$ ) provenientes do Biotério Central do Centro de Ciências Biológicas e da Saúde (CCBS) da Universidade Estadual do Oeste do Paraná (UNIOESTE). Os animais foram mantidos em biotério setorial do laboratório de Fisiologia e Biofísica do CCBS, em caixas individuais em ambiente com ciclo claro-escuro (luzes acesas as 07:00 horas e apagadas às 19:00 horas) e temperatura controlados. As ratas foram separadas em caixas individuais em grupos de 3-5 por caixa $(41 \mathrm{~cm}$ de comprimento $X 34 \mathrm{~cm}$ de largura $X 17 \mathrm{~cm}$ altura), sendo que todas receberam dieta padrão e água à vontade por todo o período experimental.

O projeto foi aprovado pelo Comitê de Ética no Uso de Animais (CEUA) da UNIOESTE (parecer 18/16 de 13/05/2016), estando de acordo com os Princípios Éticos na Experimentação Animal, adotados pelo Conselho Nacional de Controle de Experimentação Animal (CONCEA).

\section{Grupos Experimentais}

Os animais foram divididos em 4 grupos, cada grupo contendo 10 ratas, totalizando 40 ratas para o experimento.
1. Grupo Controle (CON);

2. Grupo Ligadura (LIG): onde foi induzida a doença periodontal por ligadura;

3. Grupo Ovariectomia $(\mathrm{OVX})$ : ratas submetidas à ovariectomia;

4. Grupo Ovariectomia e Ligadura (OVX+LIG): ratas submetidas à ovariectomia e onde foi induzida doença periodontal por ligadura.

\section{Indução da Doença Periodontal}

Aos 70 dias de vida os animais foram anestesiados (xilazina $0,04 \mathrm{~mL} / 100 \mathrm{~g}$ e quetamina $0,08 \mathrm{~mL} / 100 \mathrm{~g}$ ), e posicionados em mesa operatória apropriada, a qual permitiu a manutenção da abertura bucal dos ratos facilitando o acesso aos dentes da região posterior da mandíbula. Com o auxílio de uma pinça modificada e de uma sonda exploradora, foi colocado um fio de algodão número 40 ao redor do primeiro molar inferior direito. Esta ligadura atuou como irritante gengival por 30 dias, favorecendo o acúmulo de placa bacteriana e consequente desenvolvimento da doença periodontal ${ }^{17}$.

\section{Ovariectomia}

Aos 79 dias de vida, os animais foram anestesiados (xilazina $0,04 \mathrm{~mL} / 100 \mathrm{~g}$ e quetamina $0,08 \mathrm{~mL} / 100 \mathrm{~g}$ ) e posteriormente foi realizada uma incisão na pele entre a última costela e a coxa. Foi realizada uma ligadura logo abaixo da tuba uterina e o ovário foi isolado e retirado. As camadas muscular e cutânea foram suturadas separadamente. O ovário do lado oposto foi removido através do mesmo processo. Após a cirurgia, as ratas foram tratadas com pentabiótico (Fort Dodge, 0,1mL / rato, im) e analgésico (meglumina Fluxinin, Banamine; Schering-Plough, $2,5 \mathrm{mg} / \mathrm{kg}$, sc) e colocadas em gaiolas individuais.

\section{Descalcificação, processamento histológico e inclusão em blocos de parafina}

Após a eutanásia dos animais, as hemimandíbulas do lado direito obtidas foram fixadas em solução de formol a $10 \%$ por 24 horas. Após este período, foram lavadas em água corrente por 1 hora e imersas em solução de ácido fórmico preparado da seguinte forma: para cada litro de solução, $820 \mathrm{~mL}$ de água destilada e $180 \mathrm{ml}$ de ácido fórmico a 85\% (Ácido fórmico 85\% P.A., cód. 288, lote 0907252, Vetec Química Fina, Rio de Janeiro, Brasil). As peças foram mantidas na solução descalcificadora por aproximadamente 7 dias, já que a temperatura ambiente interfere no tempo de descalcificação. As peças foram avaliadas diariamente a fim de se verificar o grau esperado de descalcificação. Após este intervalo de tempo, as peças foram novamente lavadas em água corrente por 1 hora e foi feito o processamento histológico automático por aproximadamente 12 horas (Processador automático de tecidos, Leica Microsystems ${ }^{\circledR}$ TP1020, Nussloch, Alemanha), onde as peças foram imersas em formol (para fixação), álcool (para desidratação) e xilol (para diafinização ou clareamento). A partir deste, foi feita a inclusão das peças e obtenção de blocos de parafina (Parafina Purificada, cód. 1228, lote 1008459, Vetec Química Fina, Rio de Janeiro, Brasil). Os mesmos foram cortados em micrótomo semi-automático (Hestion $®$, ERM3000, DaintreeScientific, 
St. Helens, Austrália) e obtidos cortes de $7 \mu \mathrm{m}$ que foram montados em lâminas histológicas e corados pela técnica histoquímica de Hematoxilina e Eosina.

\section{Histomorfometria do osso}

A medida da crista óssea alveolar foi realizada através de um microscópio (Leica Microsystems, Suiça) acoplado a um computador, o que permitiu capturar as imagens, através do software LazEz®. Foi realizada uma medida da menor distância entre o ápice da crista óssea alveolar vestibular e a junção cemento-esmalte, utilizando um programa analisador de imagens Image Tools 3.0 (University of Texas Health Science Center, San Antonio, TX, USA ). As medidas foram repetidas uma vez por dia, em três dias diferentes, e então foi feito a média entre os valores.

\section{Análise Radiográfica}

Logo após o sacrifício, a hemimandíbula do lado esquerdo de cada animal foi retirada, fixada em formol tamponado $(\mathrm{pH} 7,2)$ durante 48 horas. As hemimandíbulas foram colocadas com o lado lingual sobre o filme radiográfico periapical (AGFA DENTUS®, Ultraspeed) e posicionados para que as cúspides vestibulares e linguais dos primeiros molares fiquem no mesmo plano vertical. Foi utilizado aparelho de raio X GE - 1000, regulado para $15 \mathrm{~mA}$, $65 \mathrm{Vp}, 18$ impulsos, distância foco/filme de 50 centímetros com incidência de raio $X$ perpendicular às peças. Para processamento dos filmes, foram utilizados revelador e fixador Kodac ${ }^{\circledR}$ no respectivo processamento de tempo/temperatura e digitalizadas através de um "scanner" para dispositivos (Polaroid Sprint Scan 35 Plus, Polaroid). As imagens digitalizadas foram analisadas em 3 medidas no programa Image Tools 3.0 (University of Texas Health Science Center, San Antonio, TX, USA) e posteriormente realizada uma média entre elas, através de uma medida linear, que percorreu a distância da junção cemento-esmalte até a crista óssea alveolar do lado mesial do primeiro molar inferior esquerdo da rata, com as medições em pixels.

\section{Análise da Expressão da Interleucina 6 (IL-6) e do Fator de} Necrose Tumoral $\alpha$ (TNF- $\alpha$ )

O tecido gengival em torno do primeiro molar inferior do lado esquerdo da hemimandíbula, submetido ou não para a colocação de ligação, foi analisada pelo ensaio imunoabsorvente de enzima (ELISA) para detectar a presença das citocinas IL-6 e TNF- $\alpha$. As proteínas totais foram extraídas das amostras do tecido gengival,

Tabela 1 - Análise histomorfométrica do primeiro molar inferior direito das ratas e análise radiográfica do lado mesial do primeiro molar inferior esquerdo das ratas da distância junção cemento-esmalte até a crista óssea alveolar de todos os grupos. Os valores representam a média \pm desvio padrão e estão expressos em pixels.

\begin{tabular}{lll}
\hline \multirow{2}{*}{ Grupos } & \multicolumn{2}{l}{ Análises } \\
\cline { 2 - 3 } & Radiográfica & Histomorfométrica \\
\hline CON & $12,76 \pm 2,04 \mathrm{~A}$ & $263,95 \pm 3,52 \mathrm{~A}$ \\
\hline LIG & $18,36 \pm 1,01 \mathrm{~B}$ & $352,77 \pm 10,95 \mathrm{~B}$ \\
\hline OVX & $16,20 \pm 0,42 \mathrm{C}$ & $295,24 \pm 2,50 \mathrm{C}$ \\
\hline OVX+LIG & $20,26 \pm 1,71 \mathrm{D}$ & $391,83 \pm 7,62 \mathrm{D}$ \\
\hline
\end{tabular}

Letras diferentes significam que os dados são estatisticamente diferentes dentro do mesmo parâmetro com $p<0.05$ e os testes utilizados foram o ANOVA e o Teste de TUKEY. utilizando um tampão de extração contendo como base um detergente de cocktail inibidor de protease. Subsequentemente, as proteínas foram quantificadas usando o método de Bradford.

\section{Análise Estatística}

Para a análise estatística, todos os valores numéricos foram expressos como média \pm desvio padrão. Em um primeiro momento foi realizado o teste de Shapiro-Wilk para avaliar a distribuição de normalidade dos dados. Após a verificação da normalidade dos dados, foram realizados o teste ANOVA e subsequentemente o teste Tukey com $\mathrm{p}<0,05$ para avaliar a diferença entre os grupos.

\section{RESULTADOS}

A análise radiográfica e histomorfométrica do osso alveolar dos primeiros molares inferiores (Tabela 1), demonstraram que no nos grupos onde foi feita a indução da doença periodontal por ligadura, houve maior perda óssea alveolar significativamente $(p<0.05)$, demonstrando a ação da periodontite induzida sobre o tecido ósseo. Como também, destaca-se o grupo OVX+LIG onde a perda óssea alveolar foi mais significativa estatisticamente $(p<0.05)$ do que nos demais grupos. Pode-se observar também que os grupos em que a periodontite não foi induzida, mas foram submetidos à ovariectomia (OVX) nota-se uma perda óssea significante $(\mathrm{p}<0.05)$ se comparados ao grupo controle, podendo sugerir uma indução da osteoporose.

A dosagem de citocinas do tecido gengival (Tabela 2), demonstraram que nos grupos onde foi realizada a cirurgia de ovariectomia, houve aumento significativo na concentração das citocinas $(p<0.05)$, em relação aos demais grupos. Destacase também o grupo OVX+LIG onde as concentrações de IL-6 e $\mathrm{TNF}-\alpha$ foram significativamente maiores quando comparadas aos demais grupos $(\mathrm{p}<0.05)$, podendo sugerir a ação da deficiência de estrógeno relacionado ao aumento de citocinas pró-inflamatórias sobre os tecidos periodontais.

\section{DISCUSSÃO}

A menopausa é um evento natural e inevitável, período o qual se define por 12 meses sem menstruação ${ }^{2,18,19}$, apresentando uma fase de transição gradual, da reprodutiva para a não reprodutiva, podendo ter seu início aos 35 anos e terminando aos 65 anos. No entanto, por volta dos 40 anos, é que as ações endócrinas sofrem alterações consideráveis, ocorrendo a exaustão dos folículos ovarianos e uma dissincronização dos sinais neuronais

Tabela 2 - Dosagem de citocinas do tecido gengival do primeiro molar inferior esquerdo das ratas. Os valores representam a média \pm desvio padrão e estão expressos em $\mathrm{pg} / \mathrm{mL}$.

\begin{tabular}{lll}
\hline \multirow{2}{*}{ Grupos } & \multicolumn{2}{l}{ Análises } \\
\cline { 2 - 3 } & TNF- $\alpha$ & IL-6 \\
\hline CON & $3,78 \pm 1,44 \mathrm{~A}$ & $22,72 \pm 16,75 \mathrm{~A}$ \\
\hline LIG & $5,97 \pm 1,05 \mathrm{~B}$ & $65,63 \pm 10,71 \mathrm{~B}$ \\
\hline OVX & $7,16 \pm 1,08 \mathrm{C}$ & $81,71 \pm 18,17 \mathrm{C}$ \\
\hline OVX+LIG & $12,24 \pm 1,19 \mathrm{D}$ & $119,16 \pm 23,07 \mathrm{D}$ \\
\hline
\end{tabular}

Letras diferentes significam que os dados são estatisticamente diferentes dentro do mesmo parâmetro com $p<0.05$ e os testes utilizados foram o ANOVA e o Teste de TUKEY. 
no hipotálamo e sistema nervoso central, a perda total da atividade folicular ovariana, acometendo a estrutura e função do ovário ${ }^{2,18,19}$.

A menopausa traz consequências biopsicossociais e faz parte do processo de envelhecimento feminino. Não sendo considerada uma doença, os sinais e sintomas associados a esse período podem ter seu tratamento através de terapêuticas especificas para aliviar tais sinais ${ }^{1}$. Esses sinais e sintomas consistem principalmente pela queda do hormônio estrógeno e podem ser caracterizados por fogachos, suores noturnos, distúrbios do sono, oscilações de humor, déficits cognitivos, ansiedade, fadiga entre outros. Em consequência a menopausa, doenças tardias podem surgir, dentre elas, doenças cardiovasculares e a osteoporose ${ }^{20}$.

A osteoporose é definida como uma doença esquelética sistêmica, caracterizada por uma diminuição da massa óssea e deterioração da microarquitetura, com consequente aumento da fragilidade óssea e maior suscetibilidade às fraturas $^{20,21}$. Embora a deficiência do estrógeno, ter sua ação antirreabsortiva do tecido ósseo bem conhecida, seu mecanismo de ação não é bem esclarecido, mas sabe-se que os hormônios sexuais têm um importante papel no crescimento ósseo e na manutenção do pico de massa óssea ${ }^{4,21-23}$. Logo justifica-se a realização deste estudo para observar a influência da deficiência de estrógeno na contribuição de um aumento na perda óssea alveolar em ratas com periodontite induzida

Em nosso estudo a indução da menopausa, através da técnica de ovariectomia demonstrou ser efetiva na diminuição da ação estrogênica, como pode-se observar, nos resultados da Tabela 1. Os resultados demonstraram um aumento significativo da perda óssea alveolar nos grupos em que a ovariectomia foi realizada em comparação ao grupo controle, com destaque maior quando observado o grupo que foi ovariectomizado recebeu a indução da periodontite experimental.

As alterações hormonais nas mulheres, a exemplo da menopausa, podem ser mediadores de inflamação dos tecidos periodontais. A interação da progesterona com o estrógeno e suas oscilações apresenta alterações clínicas no periodonto, uma vez que suas células são tecidos alvo dos hormônios esteróides $^{24}$ sendo a redução dos estrogênios séricos poderia promover maior perda óssea trabecular do que cortical, como demonstrado em nosso estudo (Tabela 1 ).

Há presença de receptores estrogênicos em osteoblastos e a principal ação desse hormônio é na reabsorção óssea. Esses receptores do estrógeno podem realizar uma ligação aos receptores dos osteoblastos e inibir a liberação de fatores estimuladores dos osteoclastos ou ainda aumentar a atividade dos fatores dos mesmos. Também, o estrógeno inibe citocinas (interleucinas 1 e 6 e TNF- $\alpha$ ), bem como fatores locais produzidos pelo osteoblasto, que estimulam a chegada dos osteoclastos nas unidades de remodelação óssea e promovem maior atividade desta linhagem celular ${ }^{4,21-23}$. Esta afirmação vai de acordo com os resultados de nosso estudo (Tabela 2), onde foi demonstrado que nos grupos onde foi realizada a cirurgia de ovariectomia houveaumento significativo na concentração de IL- 6 e TNF- $\alpha$, com destaque ainda maior quando associada a peridontite experimental, sugerindo que a deficiência de estrógeno poderia estar contribuindo para uma evolução acelerada da perda de tecido ósseo alveolar nas ratas.

A acumulação local de bactérias e de seus fatores derivados podem estimular uma reação inflamatória local na periodontite e a ativação do sistema imune inato. As células imunes secretam citocinas que promovem a maturação de osteoclastos, o que leva a um desequilíbrio no metabolismo ósseo ${ }^{25,26}$. No entanto, estes processos levam tempo para ocorrer. Portanto, as ratas do grupo OVX+ LIG apresentaram aumento de IL-6 e TNF- $\alpha$ que poderia estimular aumento da reabssorção óssea osteoclástica na deficiência de estrogénios ${ }^{26,27}$. Este conceito está de acordo com os resultados apresentados anteriormente que sugeriam alterações celulares dos linfócitos T e B, induzindo pela diminuição do estrogênio, o aumento da produção local de citocinas ativos ósseas e, finalmente, resultando na progressão de periodontite ${ }^{26,28}$.

Pallos et al. ${ }^{15}$ (2006) pesquisaram se a menopausa era um fator de risco para a periodontite. Das 46 mulheres avaliadas, entre 44 e 68 anos de idade, houve uma avaliação de parâmetros periodontais comparado aos níveis de estrógeno e da densidade óssea mineral, logo o estudo demonstrou dados que não correlacionavam a doença periodontal com a menopausa e osteoporose. Entretanto Bertulucci et al..$^{25}$ (2013) pesquisaram a mesma relação, demonstrado no estudo com 99 mulheres na pós-menopausa, na qual avaliouse a condição periodontal e comparou-a com a osteoporose pela avaliação da densidade mineral óssea. Os resultados demonstraram que o grupo de mulheres com osteoporose, apresentou maior percentual de doença periodontal e teve como conclusão que a osteoporose pode ter uma influência na condição periodontal, por haver relação entre periodontite e osteoporose em mulheres na pós-menopausa. Este estudo foi semelhante ao realizado por Mohammad e Brunsvold ${ }^{29}$ (1996), que em estudo de relação entra a osteoporose pós-menopausa e a doença periodontal, observaram-se 20 mulheres entre as idades de 50 a 75 anos, onde as condições periodontais foram comparadas a densidade óssea mineral e sugeriram que a osteoporose como doença sistêmica pode contribuir para doença periodontal, corroborando com os resultados demonstrados neste estudo (Tabela 1).

\section{CONCLUSÃO}

Dentro dos limites de nosso estudo em relação a outras análises, podemos concluir que a deficiência de estrógeno poderia contribuir, com o aumento IL-6 e TNF- $\alpha$ para uma evolução mais rápida da perda óssea alveolar em ratas ovarietomizadas, tornando-se assim um fator de risco para o desenvolvimento da periodontite.

\section{AGRADECIMENTOS}

Os autores agradecem à CAPES, Fundação Araucária e a Universidade Estadual do Oeste do Paraná (UNIOESTE) pelo suporte financeiro e ao Laboratório de Fisiologia Endócrina e Metabolismo e de Biologia Celular da Universidade Estadual do Oeste do Paraná (UNIOESTE) pelo apoio estrutural. Os autores também são agradecidos á Celeste da Rocha Paiva, técnica do Laboratório de Biologia Celular, pela assistência técnica na realização deste estudo. 


\section{REFERÊNCIAS}

01. Poli MEH, Schwanke CHA, Cruz IBM. [Menosopause in gerontology vision]. Scientia Med. 2010;2:176-84. Portuguese.

02. Wise PM, Smith MJ, Dubal DB, Wilson ME, Krainak KM, Rosewell KL. Neuroendocrine Influences and Repercussions of the Menopause. Endocrine Rev. 1999;20(3):243-8.

03. Brandão CMR, Machado GPM, Acurcio FA. [Pharmacoeconomic analysis of the osteoporosis treatment strategies for women in postmenopausal women: a systematic review]. Rev Bras Reumatol. 2012;52(6):912-37. Portuguese.

04. Amadei UA, Silveira V, Pereira AC, Carvalho Y, Rocha RF. Effect of estrogen deficiency on bone turnover and bone repair]. Bras Patol Med Lab. 2006;42(1):5-12. Portuguese.

05. Campos MLG, Corrêa MG, Velasco FG, Sallum EA, Nociti Jr $\mathrm{FH}$, Casati MZ, et al. [Estrogen deficiency and osteoporosis as current paradigms in periodontology]. R Period. 2009;19(3): 64-72. Portuguese.

06. Rivera MF, Lee JY, Aneja M, Goswami V, Liu L, Velsko IM, et al. Polimicrobial infection with major periodontal pathogens induced periodontal disease and aortic atherosclerosis in hyperlipidemic apoe null mice. Plos One 2013;8(2):1-10.

07. Sakalauskiene J, Giedrimiene D, Kubilius R, Gleiznys A, Vitkauskiene $\mathrm{A}$, Ivanauskiene $\mathrm{E}$, et al. Cytokine production by leukocytes in patients with periodontitis. Cent Eur J Med. 2014;9(6):821-9.

08. Nishimura F, Murayama Y. Periodontal inflammation and insulin resistance - lessons from obesity. J Dent Res. 2001:80(8):1690-4.

09. Orban Z, Remaley AT, Sampson M, Trajanoski Z, Chrousos GP. The differential effect of food intake and beta-adrenergic stimulation on adipose-derived hormones and cytokines in man. J Clin Endocrinol Metab. 1999;84:2126-33.

10. Vgontzas AN, Papanicolaou DA, Bixler EO, Kales A, Tyson K, Chrousos GP. Elevation of plasma cytokines in disorders of excessive daytime sleepness: role of sleep disturbance and obesity. J Clin Endocrinol Metab. 1977; 82:1313-6.

11. Yakob M, Meurman JH, Jogestrand T, Nowak J, Söder PO, Söder B. $\mathrm{C}$-reative protein in relation to early atherosclerosis and periodontitis. Clin Oral Inv. 2012;16:259-65.

12. Lopes FF, Loureiro FHF, Pereira AFV, Pereira ALA, Alves CMC. [Association between osteoporosis and periodontal disease in women post-menopause]. Rev Bras Ginecol Obst. 2008;30(8): 379-83. Portuguese.

13. Passos JSP, Gomes Filho IS, Vianna MIP, Cruz SS, Oliveira DC, Farias TS, et.al. [The influence of postmenopausal osteoporosis in periodontal condition - a literature review]. R Clin Med Biol. 2010;9(2):157-62. Portuguese.

14. Bertulucci LAB, Pereira FMBG, Oliveira AEF, Brito LMO, Lopes FF. [Periodontal disease in women in post-menopause and its relationship with osteoporosis]. Rev Brasi Ginecol Obst. 2012;34(12):563-7. Portuguese.

15. Pallos D, Ceschin A, Victor GA, Bulhões RC, Quirino MRZ. [Menopause: a risk factor for periodontal disease?]. Rev Bras Ginecol Obst. 2006;28(5):292-7. Portuguese.

16. Tanaka K, Sasaki S, Murakami K, Okubo H, Takahashi Y, Miyake Y, et al. Relationship between soy and isoflavone intake and periodontal disease: the freshmen in dietetic courses study II. BMC Public Health 2008; 8(39):1-8.

17. Nassar PO, Nassar CA, Guimarães MR, Aquino SG, Andia DC, Muscara MN, et al. Simvastatin therapy in cyclosporine A- induced alveolar bone loss in rats. J Periodontal Res. 2009; 44(4):479-88.

18. Aldrighi JM, Aldrighi CMS, Aldrighi APS. [Systemic changes in climacterium]. Rev Bras Med. 2002;59:15-21. Portuguese .

19. Matt WDW, Kauma SW, Pincus SM, Veldhuis JD, Evans WS. Characteristics of luteinizing hormone secretion in younger versus older premenopausal women. Am J Obstet Gynecol. 1998;178(3):504-10.

20. WHO-World Health Organization. Scientific group on the assessment of osteoporosis at primary health care level. Sumary Meeting Report Brussels. Belgium, 2007. [cited 2014 Feb 14]. Available at: http:// www.who.int/chp/topics/Osteoporosis.pdf.

21. Russo LAT. [Post-menopause osteoporosis: therapeutic options]. Arq Bras Endocrinol Metab. 2001;45(4):401-6. Portuguese.

22. Compston JE. Sex steroids and Bone. Physiological Rev. 2001;81(1): 419-47.

23. Ramalho AC, Lazaretti-Castro M. [Pathophysiology of involution osteoporosis]. Arq Bras Endocrinol Metab. 2006;42(6):409-14. Portuguese.

24. Santos PAT, Fortes TMV, Cabral MCB, Novaes SMA. [Influence of sexual hormones on periodontal tissues in hormonal contraceptives users]. R Period. 2008;18(1):55-63. Portuguese.

25. Di Benedetto A, Gigante I, Colucci S, Grano M. Periodontal disease: linking the primary inflammation to bone loss. Clin Dev Immunol. 2013, doi:503754: 1-7.

26. Xu XC, Chen H, Zhangi X, Zhai ZJ, Liu XQ, Zheng XY, ey al.Effects of oestrogen deficiency on the alveolar bone of rats with experimental periodontitis. Mol Med Reports 2015;12: 3494-502.

27. Miyaura C, Kusano K, Masuzawa T, Chaki O, Onoe Y, Ayoagi M, et al. Endogenous bone-resorbing factors in estrogen deficiency: cooperative effects of IL-1 and IL-6. J Bone Miner Res. 1995;10:1365-73.

28. Inagaki K, Kurosu Y, Sakano M, Sugiishi T, Yamamoto G, Noguchi $\mathrm{T}$, et al. Osteoporosis and periodontal disease in postmenopausal women: Association and mechanisms. Clin Calcium 2006;16:269-77.

29. Mohammad AR, Brunsvold M. The strength of association between systemic postmenopausal osteoporosis and periodontal disease. Int J Prosthodont. 1996:9(5):479-83.

\section{ABSTRACT}

Aim: The relation of osteoporosis on the post-menopause is controversial; however, it can be a risk factor for the periodontal disease. Therefore, the aim of this study was to evaluate the influence of the estrogen deficiency on the periodontal tissues of female ovariectomized rat with induced periodontitis. Materials and methods: Forty rats with 8 weeks of life were randomly divided into 4 groups: 1) Control group; 2) Ligature group; 3 )
Ovariectomized group; 4) Ovariectomized and ligature group; that being that all the animals were treated in a standard diet and water available all time. When reaching 70 days of life, the animals were anesthetized and a periodontal disease induction was carried, after this procedure, when reaching 79 days of life, the rats were also submitted to anesthesia and went through the ovariectomy surgery. When reaching 100 days of life, the female 
rats were sacrificed, at when the left and right hemimandible of each rat were taken for radiographic and histomorphometric analysis, as the samples of the gingival tissue to evaluate the periodontal tissue. Results: The results highlighted that the radiographic and histomorphometric analysis of the lower alveolar bone first molars of the groups in which the induction of the periodontal disease by ligature, there was higher alveolar bone loss significantly $(\mathrm{p}<0.05)$. When it comes to the relation of the cytokine concentration, the groups that were ovariectomized presented significant higher concentration when compared to the others ( $\mathrm{p}<0.05)$. Conclusion: In this way, we could conclude that the estrogen deficiency could contribute to the quicker evolution of the alveolar bone loss on female ovariectomized rats.

Keywords: Periodontitis, menopause, osteoporosis, ovariectomy.

\section{AUTOR PARA CORRESPONDÊNCIA}

Carlos Augusto Nassar

Curso de Odontologia da Universidade Estadual do Oeste do Paraná - UNIOESTE, Cascavel, Paraná, Brasil.

Rua Pernambuco, 593 - apto 504 - Centro -CEP 85810-020 -

Centro - Cascavel - Paraná - Brazil. Fone: + 554591170903

e-mail: canassar@yahoo.com 